Bulletin of the Section of Logic

Volume 45/1 (2016), pp. 17-31

http://dx.doi.org/10.18778/0138-0680.45.1.02

George Tourlakis

\title{
A NEW ARITHMETICALLY INCOMPLETE FIRST-ORDER EXTENSION OF GL ALL THEOREMS OF WHICH HAVE CUT FREE PROOFS ${ }^{1}$
}

\begin{abstract}
Reference [12] introduced a novel formula to formula translation tool ("formulators") that enables syntactic metatheoretical investigations of first-order modal logics, bypassing a need to convert them first into Gentzen style logics in order to rely on cut elimination and the subformula property. In fact, the formulator tool, as was already demonstrated in loc. cit., is applicable even to the metatheoretical study of logics such as QGL, where cut elimination is (provably, [2]) unavailable. This paper applies the formulator approach to show the independence of the axiom schema $\square A \rightarrow \square \forall x A$ of the logics $\mathrm{M}^{3}$ and $\mathrm{ML}^{3}$ of $[17,18,11,13]$. This leads to the conclusion that the two logics obtained by removing this axiom are incomplete, both with respect to their natural Kripke structures and to arithmetical interpretations. In particular, the so modified $\mathrm{ML}^{3}$ is, similarly to QGL, an arithmetically incomplete first-order extension of GL, but, unlike QGL, all its theorems have cut free proofs. We also establish here, via formulators, a stronger version of the disjunction property for GL and QGL without going through Gentzen versions of these logics (compare with the more complex proofs in $[2,8])$.

Keywords: Modal logic, GL, first-order logic, proof theory, cut elimination, reflection property, disjunction property, quantified modal logic, QGL, arithmetical completeness.
\end{abstract}

\footnotetext{
${ }^{1}$ This research was partially supported by NSERC grant No. 8250 .
} 


\section{Introduction}

Cut elimination is a standard proof-theoretic tool for propositional and first-order logics that are defined as Gentzen systems. The tool is also applicable to Hilbert style logics through a process of Gentzenisation of the logic, that is, introducing a provably equivalent sequent calculus for the original logic. Assuming that said Gentzenisation admits cut elimination, one may use the subformula property associated with this tool and derive metatheoretical results of the original Hilbert logic syntactically.

The point of departure of the present article is [12] where formulae of a Hilbert style modal logic $L$ are translated syntactically into formulae over the same language, in a manner that the translations are provable in $L$ if the pre-images are. As in loc. cit. we will call these translations "formulators".

By a judicious choice of the formulator mapping one can metaprove positive and negative results of the types "such and such a rule is, or is not, admissible in logic $L "$. This approach works directly on the original Hilbert style logic and is much simpler than the Gentzenisation approach, as was illustrated in [12] and is further exemplified here. The method is applicable toward simplifying old proofs but also toward discovering new results, such as the independence results (and the corollary incompleteness results) proved in the present article. It is important to note that the formulator method is applicable even if the Gentzenisation of the logic under study does not admit cut elimination, as is the case with the well known QGL.

The present article promotes the usefulness of the formulator approach further, by proving:

- That the axiom schema $\square A \rightarrow \square \forall x A$ of $\mathrm{M}^{3}$ and $\mathrm{ML}^{3}$ is not redundant in either logic, and, as a corollary, that the two logics obtained by removing this axiom are incomplete with respect to their natural Kripke structures and are also arithmetically incomplete.

- A strong version of the disjunction property for GL and QGL as a corollary of a result in [12], thus establishing said property without the benefit of Gentzenisation (contrast with $[2,8]$ ).

The two logics $\mathrm{M}^{3}$ and $\mathrm{ML}^{3}$ were originally developed in $[17,13]$ the former as a modal first-order extension of $\mathrm{K} 4$ and the latter as a common extension of GL and $\mathrm{M}^{3}$ (see Definitions 2.1 and 2.2). 
The motivation for introducing $\mathrm{M}^{3}$ in loc. cit. was to obtain a logic that acts as a provability logic for pure classical first-order logic in the sense that the conservation theorem holds, which in its simplest form states: "For all classical formulae $A$ and $B$, we have $A \vdash B$ classically iff $\square A \rightarrow \square B$ is provable modally", thus formalising the classical informal "-" via the modal box $\square$. This theorem was proved model-theoretically in $[17,18]$ and proof-theoretically in [11]. Since we have $\forall A \vdash A$ and $A \vdash \forall A$ classically, where " $\forall A$ " represents the universal closure of $A$ (see Section 2 for the exact definition), we see that $\vdash$ is "blind" to free variables. Correspondingly, given that the conservation theorem was the design criterion for $\mathrm{M}^{3}, \square$ in the language of this logic must be blind to free variables as well: $\square A$ is constructed so that it is a sentence for all $A$ (see the syntactic details in Section 2 and semantics in Section 5).

$\mathrm{ML}^{3}$ was introduced with two criteria in mind: one, to be a possible candidate for a first-order modal provability logic, for (arithmetised) provability in PA this time, in the style of GL. Two, like $\mathrm{M}^{3}$, to remain a provability logic for classical pure first-order logic. The former goal explains why it was chosen to be an extension of GL, just as QGL is. The conservation theorem was proved for $\mathrm{ML}^{3}$ in [13] verifying that the second design criterion was met. On the other hand, a nearly identical logic, the $\mathrm{QGL}^{b}$, was introduced in [20] and was proved to be arithmetically complete. We will endeavour to establish in a future publication the conjecture that $\mathrm{ML}^{3}$ is arithmetically complete as well, since the two logics only differ in that necessitation is admissible in $\mathrm{ML}^{3}$ but primary in $\mathrm{QGL}^{b}$.

It should be noted that the provability of the schema $\square A \rightarrow \square \forall x A$ in both $\mathrm{M}^{3}$ and $\mathrm{ML}^{3}$ was dictated in $[17,18,13]$ by the design requirement to have the conservation theorem while it was also adopted in [20] with no explicit rationale. Indeed, given that we have $A \vdash \forall x A$ classically, conservation requires that the schema is provable in each of these logics. To meet this it was sufficient to add the schema as an axiom. The present article now shows that it was also necessary.

The import of $\mathrm{ML}^{3}$ itself - besides being a provability logic for classical pure predicate logic - is in what it "can do" that QGL cannot: 
- Its Gentzenisation admits cut elimination, ${ }^{2}$ while that of QGL does not [2].

- $\mathrm{ML}^{3}$ is semantically complete with respect to finite, transitive, reverse well-founded Kripke structures - just like GL is - while QGL is not complete with respect to any class of Kripke structures [7].

Is the restriction that our $\square A$ be closed too strong? No, because on one hand, it is a natural requirement toward obtaining the conservation theorem that allows $\mathrm{ML}^{3}$ (and $\mathrm{M}^{3}$ ) to efficiently simulate classical pure first-order proofs, especially when the latter are formulated in the Equational style $[17,18]$. On the other hand, we prove in this paper that the logic $\mathrm{ML}^{2}$ - that is, $\mathrm{ML}^{3}$ with the axiom schema $\square A \rightarrow \square \forall x A$ removed - is arithmetically incomplete and therefore the first order structure has added a substantial layer of complexity making this logic behave differently, vis a vis arithmetical completeness, than its propositional restriction, GL.

This incompleteness result is significant since $\mathrm{ML}^{2}$ is essentially the "same" logic as QGL (if we set aside the difference in the syntax and semantics ${ }^{3}$ of $\square A$ between the two): they both extend GL by adding firstorder structure but do so without adding any axioms beyond those needed by the classical pure predicate calculus (without equality); and they both are arithmetically incomplete. Yet, there is also a marked dissimilarity between the two: since $\mathrm{ML}^{2}$ is a sublogic of $\mathrm{ML}^{3}$, there is a cut free proof within the Gentzen style logic GLTS $[13],{ }^{4}$ for every theorem of $\mathrm{ML}^{2}$. No similar result holds for QGL [2].

Thus $\mathrm{ML}^{2}$ provides an example of a first-order arithmetically incomplete extension of GL, all of whose theorems have proofs in a companion (but not equivalent) cut free Gentzen logic.

\section{The first-order modal logics $\mathrm{M}^{3}, \mathrm{ML}^{3}$ and QGL}

Our first-order modal alphabet uses the connectives $\rightarrow, \perp, \forall$ and $\square$ to build formulae. The remaining familiar connectives are formed via definitions. The alphabet contains no constant or function symbols but has predicates,

\footnotetext{
${ }^{2}$ Strictly speaking, the Gentzenisation introduced in [13] is cut free, but was proved that the cut rule is admissible in it.

${ }^{3}$ Semantics of QGL are covered in [7] and are not needed for the results of this paper.

${ }^{4}$ In loc. cit. GLTS is cut free by definition, but provably the cut rule is admissible. Of course, GLTS is not equivalent to $\mathrm{ML}^{2}$, since the former can also prove $\square A \rightarrow \square \forall x A$ [13].
} 
$\phi, \psi$ (with or without primes or subscripts), not including an equality predicate. As in [10], we employ two types of object variables: free, denoted by $a, b, c$ with or without primes or subscripts, and bound, denoted by $x, y, z$ with or without primes or subscripts. Thus, as was carefully defined in [11], the metalogical abbreviation " $\forall(x, a) A$ ", or simply " $\forall x A$ " if the free variable $a$ is understood from the context, stands for the expression $\forall x A[a:=x]$ - where " $a:=x$ " indicates substitution from right to left - formed by first replacing all the $a$ by the bound variable $x$ and then by prepending the string $\forall x$ to the so obtained expression. The well-known rules of formula formation will be omitted but we will elaborate on the way $\square$ is used to build new formulae:

- In the language $L_{Q G L}$ of QGL, if $A$ is a formula, then so is $\square A$. The free variables of $\square A$ are precisely the free variables of $A$.

- In the language $L_{M}$ of $\mathrm{M}^{3}$ and $\mathrm{ML}^{3}$, the expression $\square A$ is metatheoretical (textual abbreviation) and denotes the formula formed from $A$ as follows: Let $a_{j_{1}}, \ldots, a_{j_{n}}$ be all the free variables of $A$, in ascending alphabetical order. Let $x_{k_{1}}, \ldots, x_{k_{n}}$ be the first unused in $A$ bound variables, in ascending order. Form the expression $\widetilde{A}$ by substituting each occurrence of $a_{j_{i}}$ by $x_{k_{i}}$. Then the expression $\square x_{k_{1}} \ldots x_{k_{n}} \widetilde{A}$ is a formula, denoted in metanotation by $\square A .^{5}$ Thus, if $A$ has no free variables, then $x_{k_{1}} \ldots x_{k_{n}}$ is the empty string, and thus, in this case, the meta name " $\square A$ " names precisely the string formed by prepending $\square$ to $A$, i.e., the name and the named are identical strings. Clearly, $\square A$ is always closed. $A$ is the scope of the leading $\square$ and the bound variables $x_{k_{1}}, \ldots, x_{k_{n}}$ belong to this $\square$.

The first-order modal logic $\mathrm{M}^{3}$ over the language $L_{M}$ was originally introduced and investigated model-theoretically in $[17,18]$ with the goal of formalising the expression $\vdash A$ as $\square A$ for classical $A$. Its syntactic proof theory was first developed in [11] via cut elimination after proving its validity in the Gentzenisation (GTKS) of $\mathrm{M}^{3}$.

Definition 2.1 (Axioms and Rules of Inference for $\mathrm{M}^{3}$ ). The set of logical axioms of $\mathrm{M}^{3}$ is $\Lambda_{M 3} \cup \square \Lambda_{M 3}$, where $\Lambda_{M 3}$ consists of all instances of the following basic schemata:

\footnotetext{
${ }^{5}$ This description and use of $\square A$ as metanotation parallels the one in Bourbaki [4] for the meta-expression $\tau_{x} A$.
} 
(1) All tautologies

(2) $\forall x A[x] \rightarrow A[a]$

(3) $A[a] \rightarrow \forall x A[x]$, provided $a$ does not occur $A$.

(4) $\forall x(A \rightarrow B) \rightarrow \forall x A \rightarrow \forall x B$

(5) $\square(A \rightarrow B) \rightarrow \square A \rightarrow \square B$

(6) $\square A \rightarrow \square \square A$

(7) $\square A \rightarrow \square \forall x A$

The primary rules of inference are: modus ponens and "strong" ${ }^{6}$ generalisation "from $A$ infer $\forall x A$ ". The notation $\Gamma \vdash_{L} A$ in general means that $A$ is derived from hypotheses $\Gamma$ within logic $L$.

A closely related first-order logic $\mathrm{ML}^{3}$ over the same language $L_{M}$ is defined below by adding Löb's axiom (schema) $\square(\square A \rightarrow A) \rightarrow \square A$. This logic is a first-order extension of the propositional provability logic GL and was first introduced in [13], along with its Gentzenisation GLTS that was proved to admit cut elimination.

Definition 2.2 (Axioms and Rules of Inference for $\mathrm{ML}^{3}$ ). The set of logical axioms of $\mathrm{ML}^{3}$ is $\Lambda_{M L 3} \cup \square \Lambda_{M L 3},{ }^{7}$ where $\Lambda_{M L 3}$ is $\Lambda_{M 3}$ plus all instances of the Löb schema. $\mathrm{ML}^{3}$ has the same primary rules as $\mathrm{M}^{3}$.

Definition 2.3 (Partial generalisations of a formula $A$ ). The set $P G(A)$ of all partial generalisations of $A$ is defined recursively: a) $A \in P G(A)$, and b) if $B \in P G(A)$, then so are i) $\square B$ and ii) $\forall x B$, where " $x$ " stands for any bound variable. Omitting case b)i) in the recursion step we obtain $C L P G(A)$, the set of classical partial generalisations. For a set $\Gamma$, $[C L] P G(\Gamma)=\bigcup_{A \in \Gamma}[C L] P G(A)$.

Some members of $P G(A): A, \square A, \forall x A, \forall x \square A$, and $\square \forall y \square \forall z \forall x \forall x \square \forall z A$. Note. The definition does not assume a classical ( $\square$-free) language, not even in the case of $C L P G(A)$.

${ }^{6}$ Weak generalisation is usually introduced as a derived rule in first-order logics that are defined with only modus ponens as primary, cf. [5,6]. It states: if $A$ is provable with a condition on the assumptions $\Gamma$, then $\forall x A$ is also provable from $\Gamma$.

${ }^{7}$ Adding $\square \square \Lambda_{M L 3}$ to the axioms renders axiom schema $\square A \rightarrow \square \square A$ redundant, but we find it technically more convenient to not add this part in the axioms. 
Definition 2.4 (Axioms and Rules of Inference for QGL). Recall that in the language $L_{Q G L}$ the formula $\square A$ need not be closed. The set of logical axioms of QGL is $P G\left(\Lambda_{Q G L}\right)$, where $\Lambda_{Q G L}$ consists of all instances of the schemata (1)-(6) of $\mathrm{M}^{3}$ plus all instances of Löb's axiom schema. There is one primary rule of inference: modus ponens.

REMARK 2.5. Axiom schema (1) enables proof by tautological implication, that is, if $A_{1} \rightarrow A_{2} \rightarrow \ldots \rightarrow A_{n} \rightarrow X$ is a tautology, then $X$ is provable from the hypotheses $A_{1}, A_{2}, \ldots$ The propositional logic GL is, essentially, the logic over (without loss of generality) $L_{M}$ with axioms $\Lambda_{G L} \cup \square \Lambda_{G L}$, where $\Lambda_{G L}$ contains all tautologies and all instances of axiom schemata 5 , 6 , of $\mathrm{M}^{3}$, as well as those of Löb's schema.

LEMma 2.6. QGL supports the derived rule "if $\Gamma \vdash A$ and a does not occur in any formula of $\Gamma$, then $\Gamma \vdash \forall x A[a:=x]$ ".

Proof. Induction on proofs from $\Gamma$ in the manner of $[5,6]$. Basis: $A \in \Gamma$; then $a$ is not free in $A$ and we are done by (3) in the $\mathrm{M}^{3}$ axiom list, and modus ponens. $A \in P G\left(\Lambda_{Q G L}\right)$; then so is $\forall x A[a:=x]$. Induction step: We have $\Gamma \vdash B \rightarrow A$ and $\Gamma \vdash B$, for some $B$. By the Induction Hypothesis (I.H.) we have $\Gamma \vdash \forall x(B \rightarrow A)$ and $\Gamma \vdash \forall x B$. We are done by axiom schema 4 in the $\mathrm{M}^{3}$ list, and modus ponens.

Proposition 2.7 (Weak Necessitation (WN)). All three first-order logics introduced above support weak necessitation, that is, "if $\Gamma \vdash A$, then $\Gamma, \square \Gamma \vdash$ $\square$, where ' $\vdash$ ' is any one of $\vdash_{M^{3}}, \vdash_{M L^{3}}$ or $\vdash_{Q G L}$."

Proof. Induction on proofs from $\Gamma$. For $\vdash_{M^{3}}, \vdash_{M L^{3}}$ cf. $[17,13]$. Basis: $A \in \Gamma$; then $\square A \in \square \Gamma . A \in P G\left(\Lambda_{Q G L}\right)$; then $\square A \in P G\left(\Lambda_{Q G L}\right)$. Induction step: $\Gamma \vdash B$ due to $\Gamma \vdash A$ and $\Gamma \vdash A \rightarrow B$. By the I.H. $\Gamma, \square \Gamma \vdash \square A$ and $\Gamma, \square \Gamma \vdash \square(A \rightarrow B)$ and we are done by modus ponens and axiom 5 .

Note. QGL is introduced in the literature as an "extension of classical first-order logic" — albeit over the modal language $L_{Q G L}$ - obtained by including the GL modal axioms and adding strong necessitation, " $A \vdash$ $\square A$ ", as a primary rule. Since the classical part may be taken to be as in [5] where weak generalisation is a derived rule - and noting that the various definitions of QGL in the literature are silent on the precise nature of the classical part- we adopted the exact same approach for generalisation and 
necessitation: we hide them both in the axioms. In particular, it helps not to have to worry about the presence of strong generalisation when one wants to prove that weak necessitation holds for QGL.

\section{Formulators}

Definition 3.1 (Formulators [12]). A formula translator or formulator is a mapping, $\mathfrak{F}$, from the set of formulae over a modal language $L$ to itself such that:

1. $\mathfrak{F}(A)=A$ for every atomic formula $A$.

2. $\mathfrak{F}(A \rightarrow B)=\mathfrak{F}(A) \rightarrow \mathfrak{F}(B)$ for all formulae $A, B$.

3. $\mathfrak{F}(\forall x A[a:=x])=\forall x B[a:=x]$, where $B=\mathfrak{F}(A[a])$.

4. The free variables of $\mathfrak{F}(\square A)$ are among those of $\square A$.

REMARK 3.2. The formulator approach applies to the propositional cases as well by ignoring 3 in the definition. As the applications of Section 4 make clear, we do not want a formulator to commute with $\square$.

Definition 3.3. We let $C L_{M}$ stand for the "classical" sub logic of $\mathrm{M}^{3}$ or $\mathrm{ML}^{3}$, and $C L_{Q G L}$ that of QGL. $C L_{M}$ has as axioms the classical axiom schemata (1)-(4). No change to rules of inference. $C L_{Q G L}$ has as axioms $\bigcup_{A} C L P G(A)$, for each instance $A$ of schemata (1)-(4). No change to rules of inference. We also let $\mathrm{CpL}$ stand for the classical propositional logic over the language of GL, obtained by omitting the modal axioms of GL.

Theorem 3.4. If $\Gamma \vdash_{C L} A$ and $\mathfrak{F}$ is a formulator then $\mathfrak{F}(\Gamma) \vdash_{C L} \mathfrak{F}(A)$, where $\mathfrak{F}(\Gamma)=\{\mathfrak{F}(A): A \in \Gamma\}$ and " $C L$ " is an abbreviation of $C L_{X}$, where $X \in\left\{M^{3}, M L^{3}, Q G L\right\}$.

Proof. See [12]. The omitted proof, by induction on proofs, has four parts, I-IV, case IV being the induction step for an application of generalisation. Generalisation is irrelevant to the case where CL is the classical sublogic of QGL as the latter's only rule is modus ponens.

Corollary 3.5. If $\Gamma \vdash_{C p L} A$ and $\mathfrak{F}$ is a formulator then $\mathfrak{F}(\Gamma) \vdash_{C p L} \mathfrak{F}(A)$. 
Proof. As in the proof of 3.4, replacing $C L$ by $C p L$ throughout and noting that the generalisation case IV does not apply.

Well chosen formulators satisfy Theorem 3.4 and Corollary 3.5 for modal logics $L$, predicate or propositional, also when $\vdash_{C L}\left(\right.$ resp. $\left.\vdash_{C p L}\right)$ is replaced by $\vdash_{L}$. We call them "conservative" for the logic $L$. The proposition/criterion below, proved in [12], tells us how to recognise conservative formulators. In essence, it says that for a logic $L$ that has as classical sublogic the logic $C L$ (resp. $C p L$ ) it suffices to verify that $L$ proves the $\mathfrak{F}$-transforms of all modal axioms.

Proposition 3.6. Let $L$ be a first-order (resp. propositional) modal logic whose axiom set is $\Lambda_{L}=\Lambda_{C L} \cup \Omega_{L}$ (resp. $\left.\Lambda_{L}=\Lambda_{C p L} \cup \Omega_{L}\right)$, where $\Omega_{L}$ is the set of modal axioms of $L$. Suppose further that modus ponens and possibly generalisation are the only primary rules.

Given a formulator $\mathfrak{F}$, it is sufficient that $\vdash_{L} \mathfrak{F}(A)$, for all $A \in \Omega_{L}$, in order that $\mathfrak{F}$ be conservative for $L$.

Proof. Cf. loc. cit. The propositional case follows by omitting consideration of universally quantified formulae.

\section{Three Formulators of Interest}

The specific formulators defined in this section were all introduced in [12].

Definition 4.1. We let $\mathfrak{S}$ be the formulator that, for every formula $A$, satisfies $\mathfrak{S}(\square A)=\square A \wedge A$. Analogously we define $\mathfrak{G}$ by $\mathfrak{G}(\square A)=\square A \wedge \forall A$, where $\forall A$ is the universal closure of $A$.

Lemma 4.2 ([12]). $\mathfrak{G}$ is conservative for each of $M^{3}$ and $M L^{3}$.

LEMma 4.3 ([12]). $\mathfrak{S}$ is conservative for $Q G L$.

The following, with a shortened proof here, is adapted from 4.2 and 4.3 and their respective proofs, and applies in a unified manner to GL, QGL, $\mathrm{M}^{3}$ and $\mathrm{ML}^{3}$, for the latter two after the axiom schema $\square A \rightarrow \square \forall x A$ is removed. 
LEMMA 4.4. S is conservative for each of $M^{3}$ and $M L^{3}$ with the schema $\square A \rightarrow \square \forall x A$ removed, and also for $G L$ and $Q G L$.

Proof. We apply the test of 3.6. Thus we show that $\vdash_{X} \mathfrak{S}(A)$, where $X$ stands for any logic listed in the lemma.

1. $A=\square(B \rightarrow C) \rightarrow \square B \rightarrow \square C$. Then $\mathfrak{S}(A)=\square(B \rightarrow C) \wedge(B \rightarrow$ $C) \rightarrow(\square B \wedge B) \rightarrow(\square C \wedge C)$. Then $\vdash_{X} \mathfrak{S}(A)$ by tautological implication from axioms $A$ and $(B \rightarrow C) \wedge B \rightarrow C$.

2. $A=\square B \rightarrow \square \square B$. Then $\mathfrak{S}(A)=\square B \wedge B \rightarrow \square \square B \wedge \square B$. We have $\vdash_{X} \mathfrak{S}(A)$ by tautological implication from axioms $\square B \rightarrow \square B$, $\square B \wedge B \rightarrow \square B$ and $\square B \rightarrow \square \square B$.

3. Löb's axiom; it is applicable only to $\mathrm{ML}^{3}$, GL and QGL: $A=\square(\square B \rightarrow B) \rightarrow \square B$. Then $\mathfrak{S}(A)=\square(\square B \rightarrow B) \wedge(\square B \rightarrow$ $B) \rightarrow \square B \wedge B$. This is provable in all three logics by tautological implication from the axioms $A$ and $\square B \wedge(\square B \rightarrow B) \rightarrow B$.

4. $A \in \square \Lambda_{X}$, where $X \in\left\{\mathrm{GL}, \mathrm{M}^{3}, \mathrm{ML}^{3}\right\}$. Then $A=\square B$ for some $B \in \Lambda_{X}$. Thus $\mathfrak{S}(A)=\square B \wedge B$ is provable by tautological implication from axioms $B$ and $\square B$. If $X=\mathrm{QGL}$, then the corresponding (new) case is $A=\square B \in P G\left(\Lambda_{Q G L}\right)$, where $B \in P G\left(\Lambda_{Q G L}\right)$. By the obvious I.H. on the definition of $P G, B$ is provable, and so is $A$ by WN (2.7) and we are done by tautological implication.

Theorem 4.5. The axiom schema $\square A \rightarrow \square \forall x A$ of $M^{3}$ and $M L^{3}$ is not provable from the remaining axioms.

Proof. The reader may want to consult Section 5 for the semantic context. Let $L$ be either logic with the schema $\square A \rightarrow \square \forall x A$ removed. Let $A=\phi(a)$ be an atomic formula, and assume $\vdash_{L} \square \phi(a) \rightarrow \square \forall x \phi(x)$. Then

$$
\vdash_{L} \square \phi(a) \wedge \phi(a) \rightarrow \square \forall x \phi(x) \wedge \forall x \phi(x)
$$

by 4.4. We can find a Kripke structure where $\phi(a)$ is interpreted as $\mathbf{t}$ (true) at only one individual in the domain of the start world, and is $\mathbf{t}$ everywhere for all worlds reachable from the start world. Then $\square \phi(a) \wedge \phi(a)$ is satisfiable but $\square \forall x \phi(x) \wedge \forall x \phi(x)$ is not in said structure, contradicting (1) since the two logics (and their sublogics) are sound ([18, 13]). 
Definition $4.6([12])$. Let $\Delta$ be a nonempty set of formulae. We define the characteristic formulator of $\Delta$, denoted by $\mathcal{X}_{\Delta}$, as the formulator whose operation on boxed formulae is as follows:

$$
\mathcal{X}_{\Delta}(\square A)= \begin{cases}\top & \text { If } \Delta \vdash \square A \\ \perp & \text { If } \Delta \nvdash \square A\end{cases}
$$

where $\top$ abbreviates $\perp \rightarrow \perp$.

The following lemma is proved in loc. cit. We add here the logic GL in the list of those to which it applies.

Lemma 4.7. $\mathcal{X}_{\Delta}$, for any $\Delta$, is conservative for each of $M^{3}, M L^{3}, Q G L$ and $G L$.

Proposition 4.8 below, proved in [12], is a good example of the power of the characteristic formulator. It readily implies the disjunction property for all four logics, GL, QGL, $\mathrm{M}^{3}$ and $\mathrm{ML}^{3}$. It is worth repeating the very straightforward and short proof of the proposition in order to contrast it with the rather involved proofs of the disjunction property in [2] for the case of QGL and in [8] for the case of GL.

Proposition 4.8 ([12]). Let $\Sigma, \Xi$ be sets of classical formulae (i.e., $\square$ free), and suppose that $\Sigma, \square \Delta \vdash \bigvee \square \Psi \vee \bigvee \Xi$. Then $\Sigma \vdash \bigvee \Xi$ or there is some $B \in \Psi$ such that $\square \Delta \vdash \square B$, where deducibility $\vdash$ is that of any one of $M^{3}, M L^{3}, Q G L$, or $G L$.

Proof. Assume that $\square \Delta \nvdash \square B$, for all $B \in \Psi$, then, by definition, $\mathcal{X}_{\square \Delta}(\square B)$

$=\perp$, for all $B \in \Psi$. Also, since clearly $\square \Delta \vdash \square C$ for all $C \in \Delta$, we have that $\mathcal{X}_{\square \Delta}(\square C)=\top$, for all such $C^{\prime}$ 's. Thus, by 4.7 and the fact that formulators commute with Boolean connectives, we know that $\mathcal{X}_{\square \Delta}(\square \Delta), \mathcal{X}_{\square \Delta}(\Sigma) \vdash \bigvee \mathcal{X}_{\square \Delta}(\square \Psi) \vee \bigvee \mathcal{X}_{\square \Delta}(\Xi)$, or (since $\Sigma, \Xi$ are classical) $\top, \Sigma \vdash \perp \vee \bigvee \Xi$ which is the same as $\Sigma \vdash \bigvee \Xi$.

The disjunction property itself ( 4.10 below) is a corollary of theorem 7 proved in [2]. This theorem is reproduced below, as 4.9, and we offer a straightforward alternative proof using 4.8 and 4.4. By contrast, Avron uses in his proof an induction on the height of Gentzen proofs in a Gentzenisation of QGL, which have no "essential cuts". He notes, p. 939 of loc. cit., that "every provable QGL sequent has a proof without essential cuts". 
Proposition 4.9 (The pre-Disjunction Property for GL, QGL, $\mathrm{M}^{3}$ and $\left.\mathrm{ML}^{3}\right)$. Let $\Sigma, \Xi$ be two disjoint sets of atomic formulae and $\perp \notin \Sigma$. Then the derivability of the sequent

$$
\Sigma, \square A_{1}, \ldots, \square A_{k} \vdash \square B_{1}, \ldots, \square B_{m}, \Xi
$$

in the Gentzenisation of $Q G L$ (or of any one of $G L, M^{3}, M L^{3}$ ) is equivalent to that of

$$
\text { (for some } i: 1 \leq i \leq m \text { ): } \square A_{1}, A_{1}, \ldots, \square A_{k}, A_{k} \vdash B_{i}
$$

Proof. $(1) \rightarrow(2)$. The assumption precludes that $\Sigma \vdash \Xi$ is derivable. Thus, by 4.8 ,

$$
\square A_{1}, \ldots, \square A_{k} \vdash \square B_{i}
$$

is derivable, for some $i$ such that $1 \leq i \leq m$. Applying 4.4 to (3), followed by a tautological implication, we get (2) in the cases of GL or QGL.

Applying instead 4.2 to (3) we obtain, for some $i$,

$$
\square A_{1}, \forall A_{1}, \ldots, \square A_{k}, \forall A_{k} \vdash \square B_{i} \wedge \forall B_{i}
$$

in the cases of $\mathrm{M}^{3}$ or $\mathrm{ML}^{3}$. Since $A_{j}$ and $\forall A_{j}$ are inter derivable in these logics, and dropping $\square B_{i}$ to the right of $\vdash$ we obtain (2) once more.

$(2) \rightarrow(1)$. In the Gentzenisation of each of the following logics proceed as follows, recalling the inter derivability of $A_{j}$ and $\forall A_{j}$ for the first two bullets below:

- Case of $\mathrm{M}^{3}$ : Use the $\mathrm{TR}$ rule $([9,11]) \frac{\forall \Gamma, \square \Gamma \vdash A}{\Phi, \square \Gamma \vdash \square A, \Psi}$ on $(2)$.

- Case of $\mathrm{ML}^{3}$ : Use the GLR rule $([9,13]) \frac{\forall \Gamma, \square \Gamma, \square A \vdash A}{\Phi, \square \Gamma \vdash \square A, \Psi}$ on (2), preceding it with an application of weakening to correctly format the premise.

- Case of GL or QGL: Use the GLR rule $\frac{\Gamma, \square \Gamma, \square A \vdash A}{\Phi, \square \Gamma \vdash \square A, \Psi}$ on (2), preceding it with an application of weakening to correctly format the premise.

Note. Proposition 4.8 generalises 4.9 since atomic formulae are special cases of classical formulae. Moreover, 4.8 needed no Gentzenisation for 
its proof. The case for GL was originally proved in [8] by devising a proofsearch procedure.

Corollary 4.10 (Disjunction Property of QGL and GL - cf. [2, 8] - and $\left.\mathrm{M}^{3}, \mathrm{ML}^{3}\right) . \vdash_{L} \square A_{1} \vee \square A_{2} \vee \cdots \vee \square A_{k}$ iff there is an $i, 1 \leq i \leq k$, such that $\vdash_{L} A_{i}$, where $L$ is one of these four logics.

Proof. The if part is by necessitation followed by strengthening. For the only if part, we think in terms of the Gentzenisation of $L$ : So the sequent $\vdash \square A_{1}, \square A_{2}, \ldots, \square A_{k}$ is derivable. By (2) in 4.9 so is the sequent $\vdash A_{i}$ for some $i$.

\section{Incompleteness of $\mathrm{M}^{2}$ and $\mathrm{ML}^{2}$}

We name $\mathrm{M}^{2}$ and $\mathrm{ML}^{2}$ the logics obtained from $\mathrm{M}^{3}$ and $\mathrm{ML}^{3}$ respectively after removing the axiom schema $\square A \rightarrow \square \forall x A$. We will show that they are incomplete with respect to their natural Kripke structures. For the common language of these logics, the natural Kripke structures are coloured by the fact that $\square A$ is closed and by the presence or absence of Löb's axiom. These structures were originally defined in [18] in the context of $\mathrm{M}^{3}$ and in [13] for $\mathrm{ML}^{3}$. A pointed Kripke frame appropriate for $\mathrm{M}^{3}$ or $\mathrm{M}^{2}$ is a tuple $\mathcal{F}=\left(W, R, \alpha_{0}\right)$ where $W$ is a set of worlds, and $\alpha_{0} \in W$ is the start world, while $R$ is a transitive connectivity relation on $W$ for which $\alpha_{0}$ is a minimum, that is, for all $\beta \in W$, it is $\alpha_{0}=\beta \vee \alpha_{0} R \beta$.

A pointed Kripke frame appropriate for $\mathrm{ML}^{3}$ or $\mathrm{ML}^{2}$ is as above, where, moreover, the converse relation $R^{-1}$ is well-founded. ${ }^{8}$ A structure for the underlying language is a triple $\mathfrak{M}=\left(\mathcal{F},\left(M_{\beta}\right)_{\beta \in W}, \Vdash\right)$ with $\mathcal{F}$ as above, each $M_{\beta}$ - the domain of $\beta$ - being a nonempty set of individuals. $\Vdash$ is the forcing relation (forcing truth) defined, for each world $\alpha$, by recursion on closed instances of formulae. Parameters substituted for free variables are from $M_{\alpha}$ (this is in the style of Henkin's imported constants). An unspecified closed instance of $A$ is denoted by $A^{\prime}$, while $A_{a}^{\prime}$ denotes that all free variables, except $a$, were instantiated. $\alpha \Vdash A^{\prime}$ (resp. $\alpha \nVdash A^{\prime}$ ) means $A^{\prime}$ is true (resp. false) on $\alpha$.

- $\Vdash$ is defined on atomic formulae as we please (basis), but we must set $\alpha \nVdash \perp$.

\footnotetext{
${ }^{8}$ Transitivity of $R$ is intimately connected with the presence of the axiom $\square A \rightarrow \square \square A$, where the well-foundedness of $R^{-1}$ is so connected with the presence of Löb's axiom.
} 
- For any $A$ and $B, \alpha \nVdash A^{\prime} \rightarrow B^{\prime}$ iff $\alpha \Vdash A^{\prime}$ and $\alpha \nVdash B^{\prime}$.

- $\alpha \Vdash \forall x A_{a}^{\prime}[a:=x]$ iff, for all $i \in M_{a}, \alpha \Vdash A_{a}^{\prime}[a:=i]$.

- $\alpha \Vdash \square A$-note that $\square A$ is closed - iff, for all $\beta$ such that $\alpha R \beta$, we have $\beta \Vdash \forall A$.

A formula $A$ is true in a Kripke structure iff all its instances are true on the start world $\alpha_{0}$. It is known from $[18,13]$ that $\mathrm{M}^{3}$ and $\mathrm{ML}^{3}$, and therefore $\mathrm{M}^{2}$ and $\mathrm{ML}^{2}$, are sound with respect to their Kripke structures, that is, if $A$ is provable, then $\alpha_{0} \Vdash A$. However the latter two are not semantically complete since on one hand $\square A \rightarrow \square \forall x A$ is true for all the structures appropriate for $\mathrm{M}^{2}$ or $\mathrm{ML}^{2}$ (easy exercise; cf. loc. cit.), but not provable by 4.5 .

It is easy to see that $\mathrm{ML}^{2}$ is not arithmetically complete either, as it follows from 4.5 and the fact that the arithmetical interpretation of $\square A \rightarrow \square \forall x A$ is $\operatorname{Pr}\left(\left\ulcorner A^{*}\right\urcorner\right) \rightarrow \operatorname{Pr}\left(\left\ulcorner\forall x A^{*}\right\urcorner\right)$, but this schema is provable in PA. Here is a very high-level outline of the why: This PA-schema captures the meta statement "if $\vdash_{P A} A^{*}$, then $\vdash_{P A} \forall x A^{*}$ ". Now, informally, $\operatorname{Pr}(x)$ is the $\Sigma_{1}$ formula $\exists y \operatorname{Proof}(y, x)$ that says " $x$ is the Gödel number of a formula that appears in a PA-proof with Gödel number $y$ ", where Proof $(y, x)$ is a $\Sigma_{0}$ formula. Machinery in either of $[15,16]$ can be used straightforwardly to prove, for any formula $B$ in the language of PA, that $\vdash_{P A}$ $\operatorname{Proof}(y,\ulcorner B\urcorner) \rightarrow \operatorname{Proof}(y \frown\ulcorner\forall x B\urcorner,\ulcorner\forall x B\urcorner)^{9}$ and that is because this is a true $\Sigma_{0}$ formula. Thus, $\vdash_{P A} \operatorname{Proof}(y,\ulcorner B\urcorner) \rightarrow \exists z \operatorname{Proof}(z,\ulcorner\forall x B\urcorner)$. Using the " $\exists$-introduction rule" $([14,16])$ we get $\vdash_{P A} \exists y \operatorname{Proof}(y,\ulcorner B\urcorner) \rightarrow$ $\exists z \operatorname{Proof}(z,\ulcorner\forall x B\urcorner)$.

\section{References}

[1] S. Artemov and G. Dzhaparidze, Finite Kripke Models and Predicate Logics of Provability, J. of Symb. Logic 55 (1990), no. 3, pp. 1090-1098.

[2] A. Avron, On modal systems having arithmetical interpretations, J. of Symb. Logic 49 (1984), no. 3, pp. 935-942.

[3] G. Boolos, The Logic of Provability, Cambridge University Press, Cambridge, 1993.

[4] N. Bourbaki, Éléments de Mathématique; Théorie des Ensembles, Hermann, Paris, 1966.

9 "ح" denotes concatenation. 
[5] H. B. Enderton, A mathematical introduction to logic, Academic Press, New York, 1972.

[6] F. Kibedi and G. Tourlakis, A modal extension of weak generalisation predicate logic, Logic Journal of the IGPL 14 (2006), no. 4, pp. 591621.

[7] F. Montagna, The predicate modal logic of provability, Notre Dame J. of Formal Logic 25 (1984), pp. 179-189.

[8] G. Sambin and S. Valentini, A Modal Sequent Calculus for a Fragment of Arithmetic, Studia Logica 39 (1980), no. 2/3, pp. 245-256.

[9] , The Modal Logic of Provability. The Sequential Approach, Journal of Philosophical Logic 11 (1982), no. 3, pp. 311-342.

[10] K. Schütte, Proof Theory, Springer-Verlag, New York, 1977.

[11] Y. Schwartz and G. Tourlakis, On the proof-theory of two formalisations of modal first-order logic, Studia Logica 96 (2010), no. 3, pp. 349-373.

[12] _ A Proof Theoretic Tool for First-Order Modal Logic, Bulletin of the Section of Logic 42 (2013), no. 3-4, pp. 93-110.

[13] On the Proof-Theory of a First-Order Version of GL, J. of Logic and Logical Philosophy 23 (2014), no. 3, pp. 329-363.

[14] J. R. Shoenfield, Mathematical Logic, Addison-Wesley, Reading, Massachusetts, 1967.

[15] C. Smoryński, Self-Reference and Modal Logic, Springer-Verlag, New York, 1985.

[16] G. Tourlakis, Lectures in Logic and Set Theory; Volume 1: Mathematical Logic, Cambridge University Press, Cambridge, 2003.

[17] G. Tourlakis and F. Kibedi, A modal extension of first order classical logicPart I, Bulletin of the Section of Logic 32 (2003), no. 4, pp. 165-178.

[18] _ A modal extension of first order classical logic-Part II, Bulletin of the Section of Logic 33 (2004), no. 1, pp. 1-10.

[19] V. A. Vardanyan, On the predicate logic of provability, "Cybernetics", Academy of Sciences of the USSR (in Russian) (1985).

[20] R. E. Yavorsky, On arithmetical completeness of first-order logics, Advances in Modal Logic (F. Wolter H. Wansing M. de Rijke and M. Zakharyaschev, ed.), vol. 3, CSLI Publications, Stanford University, Stanford, USA, 2001, pp. 1-16. 